\title{
Stimulus-determined discrimination mechanisms for color search
}

\author{
ELIZABETH S. OLDS, WILLIAM B. COWAN, and PIERRE JOLICOEUR \\ University of Waterloo, Waterloo, Ontario, Canada
}

\begin{abstract}
Visual attention can be goal driven, stimulus driven, or a combination of the two. Here we report evidence for an unexpectedly stimulus-driven component of visual search for a target defined by color. Observers demonstrated a surprisingly cost-free ability to incorporate multiple classifiers in search for a target of one color from among distractors of other colors. A target color was presented among distractors that could change from trial to trial (intermixed presentation) or that remained constant across all trials in a block (blocked presentation). For blocked presentation, a single search classifier (a mechanism that segregates the target from distractors in color space) could be adopted, whereas for intermixed presentation different classifiers had to be used when the distractor colors changed. The benefit of blocked presentation was very small, suggesting that the appropriate classifier was determined very quickly in trials for which the classifier changed. The results suggest that the stimulus-driven activation of an appropriate stimulus classifier can be very efficient.
\end{abstract}

We sought to understand the process by which skilled observers can rapidly find a target presented among different distractor items. One important characterization of visual search is the extent to which it is goal driven or stimulus driven - that is, guided by intentions or guided by visual input from the world. The research reported here investigated the stimulus-driven component of search by manipulating stimulus uncertainty.

To estimate the cost of a manipulation, we categorize search performance based on response time (RT) patterns (see Treisman \& Gelade, 1980). In pop-out search, RT for correct responses does not depend on the number of distractors present; in difficult search, RT rises sharply with the number of distractors. Some relationships between target and distractor features afford pop-out search; other relationships produce difficult search.

The experiment requires the observer to search for a colored target disk from among colored distractor disks. We choose color as the feature relevant for the task because it has a well-accepted quantitative metric. The metric is based on a three-dimensional feature space (with two dimensions representing chromatic hue and saturation and the third dimension representing intensity; Wyszecki $\&$ Stiles, 1982). Every visually distinct color corresponds to a distinct point in the space. This space is useful for the characterization of target-distractor relationships. In a search task using three distractor colors that are not collinear in color space, the points representing these col-

\footnotetext{
This research was funded by a Human Frontier Science Program grant awarded to W.B.C. and P.J. The authors thank Kyle Cave, Michael D'Zmura, Steven Yantis, and an anonymous reviewer, for comments on the manuscript. Correspondence should be addressed to E. Olds, Department of Psychology, Wilfrid Laurier University, 75 University Avenue West, Waterloo, ON N2L 3CS Canada (e-mail: esolds@cg1. uwaterloo.ca).
}

ors define a triangle lying in a unique plane. If the target color lies off the plane or in the plane and outside the triangle, then it is possible to draw a single plane, or classifier, that separates all distractor colors from the target color. This configuration of colors is called linearly separable (Figures 1 and $2 a$ ). When the target is contained within the triangle defined by the distractors, no single plane can separate it from all distractors simultaneously, so it is not linearly separable (Figure $2 b$ ). ${ }^{1}$

Bauer, Jolicoeur, and Cowan (1996b) (following D'Zmura, 1991) investigated linear separability in color search, reducing the complexity of the problem by using a two-dimensional color space (constant luminance) rather than a three-dimensional color space. They found pop-out search for linearly separable displays and difficult search for displays that were not linearly separable. Bauer et al. (1996b) suggested that a linear separator mechanism, operating in color space, is responsible for pop-out search when target and distractors are linearly separable. The experiment described in this paper investigated the possibility that the mechanisms responsible for easy search associated with linearly separable configurations operate in a largely bottom-up fashion.

One can investigate the extent to which visual search is stimulus driven (bottom-up) by manipulating stimulus certainty. This can be done by controlling whether the observer knows or does not know which of three pairs of distractor colors will appear on the next trial, the target color remaining constant throughout the experiment. Figure $2 b$ shows a schematic diagram of the locations of the distractor colors to illustrate color relationships in the stimuli. The three distractor colors surround the target, which is never linearly separable from all three distractor colors presented together. However, it is linearly separable from every pair of distractors. Easy search is expected for blocked presentation because the observer knows which 


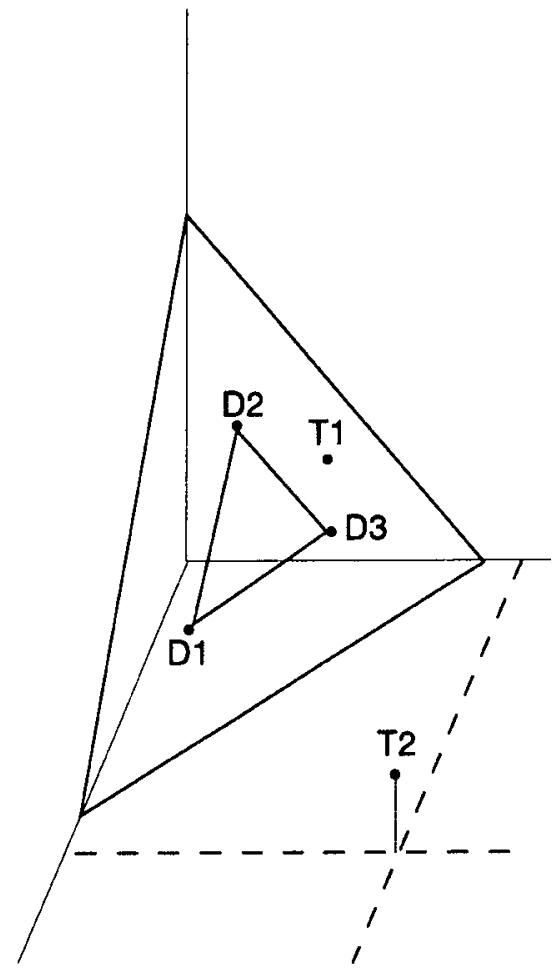

Figure 1. Illustration of three-dimensional color space, with three distractor colors D1, D2, and D3. A target of a fourth color $T 1$ lies in the plane defined by the distractor colors but not within the triangle defined by them. It can be separated from the distractors by any plane lying between $T 1$ and the D1-D2-D3 triangle-for example, a plane perpendicular to the plane defined by the distractors. A target of color $\mathrm{T} 2$ that lies outside the plane defined by the three distractors can be separated from them by, for example, a plane parallel to the triangle that falls between it and point $T 2$.

distractor pair will be used on the next trial and can therefore preselect a linear classifier. For intermixed presentation, on the other hand, it is impossible to preselect the classifier needed for the distractor colors that will actually appear. The target on each trial is linearly separable from the distractors, but only once the trial has begun can the observer know which linear separator to use. We found the cost of this uncertainty to be surprisingly low.

\section{METHOD}

To assess the effect of stimulus uncertainty, we had adults with normal color vision report whether or not a target stimulus appeared among a set of distractor stimuli. We assessed search performance using RT. Like Bauer et al. (1996b), we used equiluminant target, distractor, and background colors. We presented varying numbers of distractors of two or three colors on each trial. The number of actual distractor items $(7,16$, or 36$)$ intermixed across trials within every block. A target appeared on exactly half the trials.

The first step in our experiment was to find appropriate distractor colors, given the target color. The three colors of distractors, when presented simultaneously with the target, have to produce dif- ficult search; pairs of the distractor colors have to produce pop-out search. By linear separability, a triangular configuration of distractors that surrounds the target can produce just this pattern of results. For each observer, we chose a target-distractor distance that maximized the difference between search ease in the distractor-pair case and the distractor-triple case.

By varying the blocking of trials with different combinations of distractor colors, we can control whether or not the observer knows in advance of stimulus presentation which of three pairs of distractors would appear. In blocked presentation, all the trials of a block have the same pair of distractor colors (D1 and D2 in Condition $B_{12}$, or D1 and D3 in Condition $\mathrm{B}_{13}$, or D2 and D3 in Condition $\mathrm{B}_{23}$; see Figure 3 ). Thus the observer always knows which distractor colors will appear, although not the actual number of distractor items. In intermixed presentation, trials with the three different distractor color pairs are randomly intermixed. For these trials, the observer does not know which distractor pair will appear until stimulus onset.

In addition to the main measurements of performance in blocked and intermixed presentation of the three distractor pairs, we tested observers in two control conditions where each trial contained three distractor colors-a distractor triple. In one $\left(\mathrm{B}_{123}\right)$, the observer searched for $\mathrm{T}$ in distractors D1, D2, and D3, with target and distractors chosen so that search would be difficult. The other control condition $\left(\mathrm{B}_{124}\right)$ was a probe for the effects of heterogeneity, using a fourth color of distractor. The observer searched for $T$ in distractors D1, D2, and D4, where T was linearly separable from D1, D2, and D4 (Figure 3). Easy search was expected in this condition-otherwise, it could be argued that difficult search for T in D1, D2, and D3 was due not to lack of linear separability but to distractor heterogeneity (Duncan \& Humphreys, 1989; see also Bauer, Jolicoeur, \& Cowan, 1996a). Were distractor heterogeneity (defined as the number of distractor colors) responsible for difficult search in Condition $B_{123}$, difficult search would be found in Condition $B_{124}$ as well.

\section{Predictions}

Given the target color, we chose the distractor colors for each observer so that search in individually presented distractor color pairs would be easy (in blocked presentation). That is, the slope of the function relating RT to set size (the number of items in the display) would be small. Given these stimulus colors, we measured RT in intermixed blocks, where the target-distractor relationship was unpredictable. The pattern of data for intermixed presentation could have the following relationships to the pattern of data for blocked presentation:

1. No difference. Intermixed presentation could yield identical performance to that yielded by blocked presentation. We would infer no cost of intermixing. This would mean that multiple classifiers could be held ready or that the activation of classifiers was instantaneous.

2. Intercept change. Intermixed presentation could yield pop-out search with a low set-size function slope, similar to blocked presentation, but we might find a difference in absolute RT between the two. We would interpret the constant cost incurred by the intermixing as the time required to activate a classifier.

3. Slope change. Intermixed presentation could yield difficult search, contrasted with the easy search found for blocked presentation; this difference would manifest itself as an increase in the slope of the set-size function for intermixed presentation, relative to blocked presentation. A change in slope would indicate that if the required classifier is not consistent across trials, this variability is so jarring that difficult search is necessary even though the target on each trial is linearly separable from the distractors.

\section{Observers}

Two observers, E.O. (female, 28 years old, author) and S.L. (female, 21 years old, naive to the purposes of the experiment), had normal color vision by standard measures. ${ }^{2}$ 

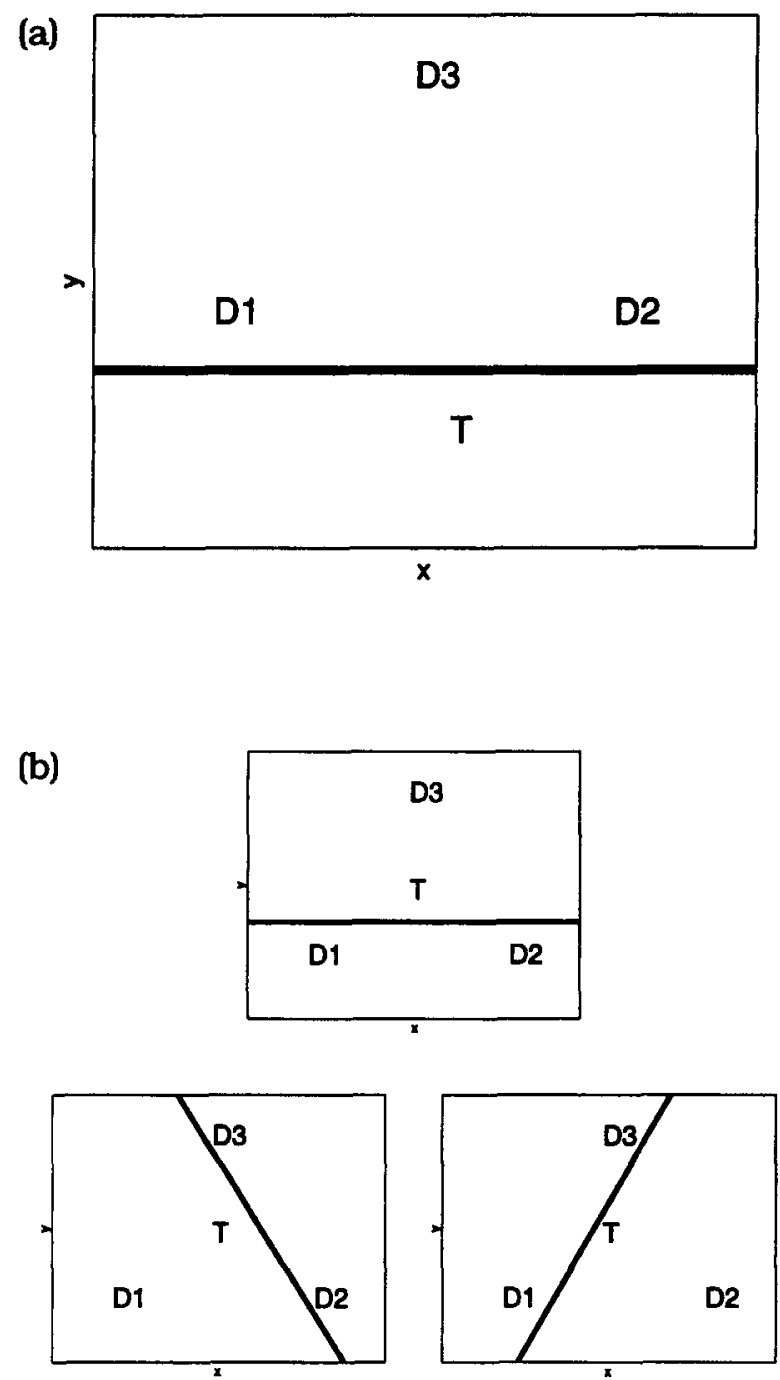

Figure 2. Here we show a two-dimensional slice through threedimensional color space in the plane defined by the three distractor colors. (a) The target $T$ is linearly separable from the distractors. (b) The target $T$ is not linearly separable from the distractors; three attempted classifiers are shown. Note that each classifier does separate the target from a pair of distractors.

\section{Equipment}

Testing was carried out on a Macintosh PowerMac 6100 computer using MATLAB software and Brainard's (1997) Psychophysics Toolbox routines. The coordinates of the colors were set using a Minolta Chroma Meter CS- 100 .

\section{Selection of Color Stimuli}

To find three appropriate distractor colors for each observer, we had to fulfill several conditions. For simplicity we chose the distractor colors to be roughly equally perceptually different from the target. Thus we chose a value for target-distractor distance (in CIELuv) that was the same for the distance between $\mathrm{T}$ and $\mathrm{D} 1$, between $T$ and D2, and between $T$ and D3. In addition, we chose to constrain the angles produced by the lines joining target to distractors to be equally spaced in $u v$ space ( $120^{\circ}$ apart; see Figure $3 \mathrm{~b}$ ).

Finally, the comparison between blocked and intermixed presentation is interesting only if the following two conditions are met.
(1) When all three distractor colors are presented with the target, search must be difficult; and (2) when any one of the three pairs is presented in blocked style with the target, search must be easy. By linear separability these two conditions should generally be true, but we sought to find a target-distractor distance that would in fact maximize the difference between search in all the three distractors and search in pairs of distractors (blocked presentation).

Our working heuristic for sufficiently attaining Conditions 1 and 2 was as follows. We measured search performance at several different target-distractor distances in CIELUv space (ranging at first from indiscriminable colors to very different colors and then narrowing this range and sampling more finely), and we chose the distractor set that maximized the following ratio:

ratio $=$

$$
\frac{\operatorname{median}\left(\mathrm{RT}_{\mathrm{D} 1 \mathrm{D} 2 \mathrm{D} 3}\right)}{\max \left[\operatorname{median}\left(\mathrm{RT}_{\mathrm{D} 1 \mathrm{D} 2}\right), \operatorname{median}\left(\mathrm{RT}_{\mathrm{D} 1 \mathrm{D} 3}\right), \operatorname{median}\left(\mathrm{RT}_{\mathrm{D} 2 \mathrm{D} 3}\right)\right]}
$$

where median( $\left.\mathrm{RT}_{\mathrm{D}_{1 \mathrm{D} 2 \mathrm{D} 3}}\right)$ is the median RT to find the target in 36 items with distractors $\mathrm{D} 1, \mathrm{D} 2$, and $\mathrm{D} 3$, medianRT $\mathrm{D}_{\mathrm{D} 1 \mathrm{D} 2}$ is the median RT to find the target in 36 items with distractors D1 and D2 only, medianRT $_{\mathrm{DID} 3}$ is the median RT to find the target in 36 items with distractors D1 and D3, and medianRT $\mathrm{D} 2 \mathrm{D} 3_{3}$ is the median RT to find the target in 36 items with distractors D2 and D3. It should be noted that we were not testing the linear separability theory in this selection method (for tests and discussion of the theory, see Bauer et al., 1996a). Instead, we sought colors that, for a given observer, allowed us to best test the flexibility of search mechanisms.

\section{Stimuli}

The experimental stimuli were colored disks measuring $8 \mathrm{~mm}$ in diameter on the screen. They subtended approximately $0.75^{\circ}$ of visual angle at the observer's viewing distance $(60 \mathrm{~cm})$. They were presented on a near-gray background. The disks and background had a luminance of $25 \mathrm{~cd} / \mathrm{m}^{2}$. (See Table 1 and Figure 3 for the color coordinates.)

We used CIELuv space, rather than chromaticity space, for actual stimulus color selection (see also Bauer et al., 1996b). CIELuv space is a commonly used and accepted color space that is a transformation of chromaticity space. Distance in CIELUv space corresponds roughly to perceived color difference. The perceptual uniformity afforded by this metric is useful for controlling the distances between target and distractors.

\section{Design}

The experimental trials involved one target stimulus (or none) and up to three colors of distractor stimuli. The number of distractors varied from trial to trial so that the total number of stimuli on the screen was 7,16 , or 36 on each trial. If a target was present, it replaced one of the distractors. This replacement occurred so that even when there were only seven stimuli in the display, there were still at least two of each distractor color.

In Conditions $\mathrm{B}_{12}, \mathrm{~B}_{13}$, and $\mathrm{B}_{23}$, the target was presented along with distractors of two colors only. This distractor pair consisted of distractor colors D1 and D2 for Condition $\mathrm{B}_{12}$; it consisted of distractor colors D1 and D3 for Condition $\mathrm{B}_{13}$; it consisted of distractor colors D2 and D3 for Condition $\mathrm{B}_{23}$.

In Condition $M_{12-13-23}$ (with Subconditions $M_{12}, M_{13}$, and $M_{23}$ ), all three pairs of distractors from Conditions $B_{12}, B_{13}$, and $B_{23}$ were presented intermixed. That is, trials containing distractors D1 and D2, trials containing distractors D1 and D3, and trials containing distractors D2 and D3 were presented in random order within the experimental session.

Distractor colors D1, D2, and D3 were presented on each trial in Condition $B_{123}$. Condition $B_{124}$ presented observers with three distractor colors that did not encircle the target in color space. These were D1, D2, and a new distractor color, D4 (Figure 3).

The target and distractor disks appeared at various locations on a (invisible) $6 \times 6$ grid. This grid of possible locations was ran- 
(a)

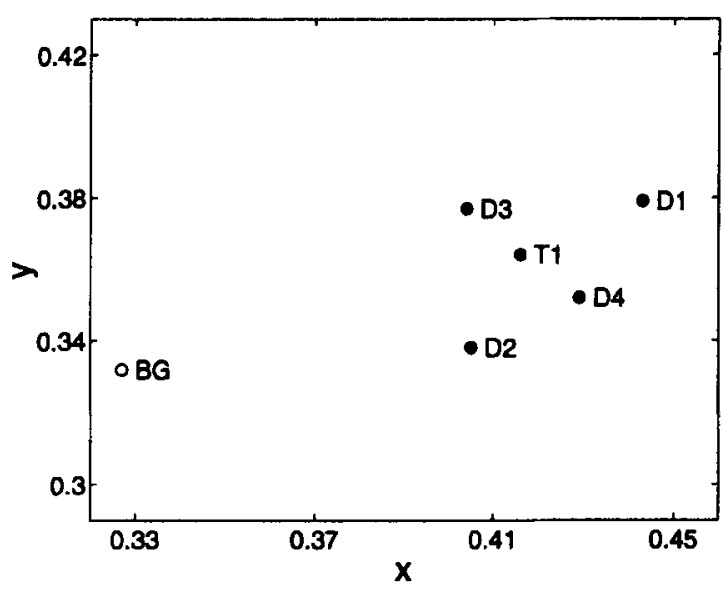

(b)

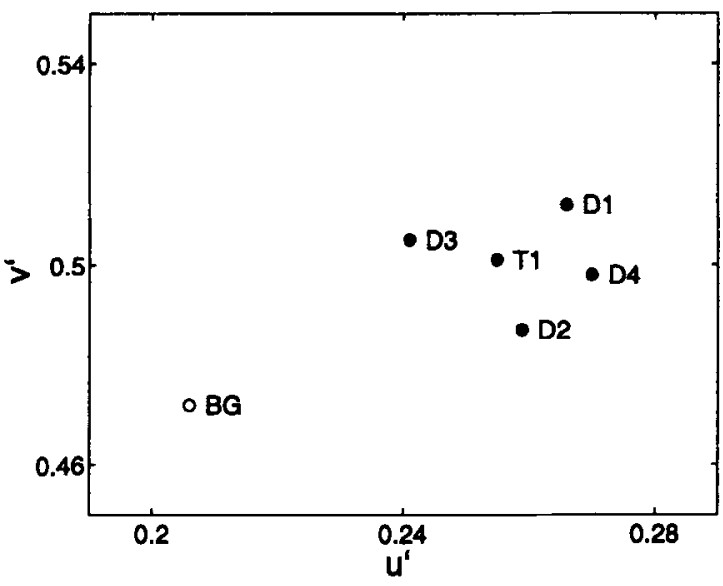

Figure 3. Stimulus colors for Observer E.O., plotted in (a) Yxy chromaticity and (b) CIELuv color spaces.

domly perturbed on every trial so that each potential disk location moved up to $\pm 0.11^{\circ}$ of visual angle in the horizontal and vertical directions. The trials were created so that for any set size, if the target appeared, it could appear at any of the possible distractor positions, except for the four corners. This resulted in 32 possible target positions, which were crossed with present/absent and set size (7, $16,36)$. The resulting number of trials was $192(32 \times 2 \times 3)$, for Conditions $B_{12}, B_{13}, B_{23}, B_{123}$, and $B_{124}$. Condition $M_{12-13-23}$ required three times as many trials because it contained, in intermixed order, all the trials from Conditions $\mathrm{B}_{12}, \mathrm{~B}_{13}$, and $\mathrm{B}_{23}$.

The complete set of 192 (or 576) trials was in fact presented in four subsets of 48 (144) trials. This allowed the ordering of experimental conditions to be more fully counterbalanced. Each subset of 48 (144) trials contained equal numbers of present and absent trials, and trials of each set size. A pseudorandom ordering of the trials prevented runs of more than three consecutive trials with the same set size or trial type (present/absent) and runs of more than four consecutive trials with the same target position.

\section{Procedure}

The observer, seated $60 \mathrm{~cm}$ from the monitor, adapted for several minutes in the dimly illuminated experimental room before testing began. In each session, the different experimental conditions were presented in a random order. A display screen at the beginning of each block specified the target color and the colors of the distractors. Each block began with 30 practice trials. The task instructions emphasized both speed and accuracy.

In a trial, the observer saw first a fixation stimulus, which was a plus sign ( + ) for the first trial. On subsequent trials, the fixation was $a+$ following a correct response on the previous trial, and a minus sign ( - ) following an error. The fixation point was presented for $400 \mathrm{msec}$, after which the screen was blank for $400 \mathrm{msec}$. Next the stimulus array was displayed on the screen until the observer responded, pressing the " $f$ " key to indicate that the target was present or the " $\mathrm{j}$ " key to indicate that the target was not present.

\section{RESULTS}

A recursive outlier screening procedure, described in Van Selst and Jolicoeur (1994), eliminated 24/1,454 correct trials based on RTs (1.7\%) for Observer E.O. and $44 / 4,450$ correct trials (1\%) for Observer S.L. Analyses are reported here for the data with the outliers removed; the results were similar with the outliers left in. Separate analyses of variance (ANOVAs) were performed on the data for each observer. Individual trials were treated as the basic unit of analysis. These analyses allowed us to determine whether the effects were significant for each observer (i.e., if they are likely to replicate, should that observer be tested again under similar conditions).

\section{Observer E.O.}

See Table 2 for the parameters of the functions relating RT to set size. See Figure 4 for the plots of RT and errors versus set size, for the blocked and intermixed conditions; see Figure 5 for the data from Condition $\mathrm{B}_{123}$ (not separable) and Condition $\mathrm{B}_{124}$ (separable).

There were six experimental conditions: $\mathrm{B}_{12}, \mathrm{~B}_{13}, \mathrm{~B}_{23}$, $\mathbf{M}_{12-13-23}, \mathbf{B}_{123}$, and $\mathbf{B}_{124}$. The most important analyses are those of correct responses to target-present trials ("hits"); we restrict our discussion to these data, but data for target-absent trials are included in the figures as well. First, RTs for correct responses to target-present trials were entered into an ANOVA with condition and set size as the main factors. Overall, experimental condition had a significant effect on hit RT $\left[F(5,685)=31.87, M S_{\mathrm{e}}=50,258\right.$,

Table 1

Chromaticity ( $x y)$ and CIELuv (uv) Stimulus Coordinates for Observers E.O. and S.L.

\begin{tabular}{llcc}
\hline \multicolumn{1}{c}{ Observer } & & $x, y$ & \multicolumn{2}{c}{$u, v$} \\
\hline E.O. \& S.L. & bg & $0.327,0.332$ & $0.206,0.472$ \\
& T & $0.416,0.364$ & $0.255,0.501$ \\
E.O. & D1 & $0.443,0.379$ & $0.266,0.512$ \\
& D2 & $0.405,0.338$ & $0.259,0.487$ \\
& D3 & $0.404,0.377$ & $0.241,0.505$ \\
S.L. & D4 & $0.429,0.352$ & $0.270,0.498$ \\
& D1 & $0.442,0.379$ & $0.265,0.512$ \\
& D2 & $0.404,0.338$ & $0.259,0.487$ \\
& D3 & $0.403,0.377$ & $0.240,0.505$ \\
& D4 & $0.428,0.352$ & $0.269,0.497$ \\
\hline
\end{tabular}

Note $-Y=25 \mathrm{~cd} / \mathrm{m}^{2}$ and $L=100$. 
Table 2

Slopes (Milliseconds/Item) and Intercepts (Milliseconds) for the Fitted Functions Relating Reaction Time to Set Size for Each Experimental Condition (Observers E.O. and S.L.)

\begin{tabular}{llllllc}
\hline & & \multicolumn{2}{c}{ E.O. } & & \multicolumn{2}{c}{ S.L. } \\
\cline { 7 - 8 } \cline { 7 - 8 } Distractor & Condition & Slope & Intercept & & Slope & Intercept \\
\hline D1\&D2, D1\&D3, or D2\&D3 & $\mathrm{B}_{12}, \mathrm{~B}_{13}$, or $\mathrm{B}_{23}$ & 6.5 & 568 & & 9.3 & 606 \\
D1\&D2, D1\&D3, or D2\&D3 & $\mathrm{M}_{12-13-23}$ & 6.7 & 581 & & 8.5 & 667 \\
D1, D2, \& D3 & $\mathrm{B}_{123}$ & 30.2 & 411 & & 21.7 & 601 \\
D1, D2, \& D4 & $\mathrm{B}_{124}$ & 6.9 & 567 & & 13.5 & 656 \\
\hline
\end{tabular}

$p<.0001]$, as did set size $\left[F(2,685)=86.12, M S_{\mathrm{e}}=\right.$ $50,258, p<.0001]$; condition and set size interacted as well $\left[F(10,685)=15.62, M S_{\mathrm{e}}=50,258, p<.0001\right]$.

Our main comparison was between blocked and intermixed presentation (in the relevant conditions, $\mathrm{B}_{12}, \mathrm{~B}_{13}$, $B_{23}$, and $M_{12-13-23}$. The RTs for hit responses were entered into an ANOVA with blocked/intermixed, distractor pair, and set size as the main variables. Because distractor pair did not interact with blocked/intermixed $\left[F(2,504)=1.69, M S_{\mathrm{e}}=28,027, p>.18\right]$, we collapsed across distractor pair for the following calculations of the main effect of blocked versus intermixed. In addition, because the interaction of blocked/intermixed, distractor pair, and set size was not significant $[F(4,504)=$ $\left..40, M S_{\mathrm{e}}=28,027, p>.8\right]$, we collapsed across distractor pair for our calculation of slope differences between blocked and intermixed presentation. (In addition, we examined the data separated by distractor pair and did not discover anything new.) Collapsing across distractor pairs, there was no main effect on RT (in the relevant conditions, $\mathrm{B}_{12}, \mathrm{~B}_{13}, \mathrm{~B}_{23}$, and $\mathrm{M}_{12-13-23}$ ) of blocked/intermixed $\left[F(1,516)=2.41, M S_{\mathrm{e}}=32,520, p>.1\right]$ and no interaction of blocked/intermixed with number of items $[F(2,516)=$ $\left.1.92, M S_{\mathrm{e}}=32,520, p>.1\right]$. The difference between RTs in the blocked and intermixed conditions was $29 \mathrm{msec}$. A main effect larger than $31 \mathrm{msec}$ would have been significant. ${ }^{3}$ By this analysis, if any effect exists, it is quite small.

Individually presented pairs from the set of three distractors (in Conditions $\mathrm{B}_{12}, \mathrm{~B}_{13}$, and $\mathrm{B}_{23}$ ) produced faster search than did the triplet of distractors presented simultaneously in Condition $\mathrm{B}_{123}[F(1,346)=90.63$, $\left.M S_{\mathrm{e}}=71,254, p<.0001\right]$; the interaction with set size was significant as well $\left[F(2,346)=45.91, M S_{\mathrm{e}}=71,254\right.$, $p<.0001]$. However, the difficulty of $\mathrm{B}_{123}$ search was not due to the number of distractor colors: $\mathrm{B}_{124}$ search was much faster than $\mathrm{B}_{123}$ search $\left[F(1,175)=37.34, M S_{\mathrm{e}}=\right.$ $108,078, p<.0001]$; the interaction with set size was also significant $\left[F(2,175)=17.09, M S_{\mathrm{e}}=108,078, p<.0001\right]$.

\section{Observer S.L.}

See Table 2 for the parameters of the function relating RT to set size. See Figure 6 for the plot of RT and errors versus set size, for the blocked versus intermixed conditions; see Figure 7 for the data from Conditions $B_{123}$ and $\mathrm{B}_{124}$.

RTs for hit responses were entered into an ANOVA with condition and set size as the main factors. Condition had a significant effect on RT $\left[F(5,2128)=49.62, M S_{\mathrm{e}}=\right.$ $94,956, p<.0001]$, as did set size $[F(2,2128)=193.20$, $\left.M S_{\mathrm{e}}=94,956, p<.0001\right]$; the interaction of condition and set size was also significant $[F(10,2128)=10.86$, $\left.M S_{\mathrm{e}}=94,956, p<.0001\right]$.

Our main comparison was between blocked and intermixed presentation (in the relevant conditions, $B_{12}, B_{13}$, $B_{23}$, and $M_{12-13-23}$ ). The RTs for hit responses were entered into an ANOVA with blocked/intermixed, distractor pair, and set size as the main variables. Because distractor pair did not interact with blocked/intermixed $[F(2,1596)=$ $\left.1.26, M S_{\mathrm{e}}=66,876, p>.2\right]$, we collapsed across distractor pair for the following calculations of the main effect of blocked versus intermixed. In addition, because the interaction of blocked/intermixed, distractor pair, and set
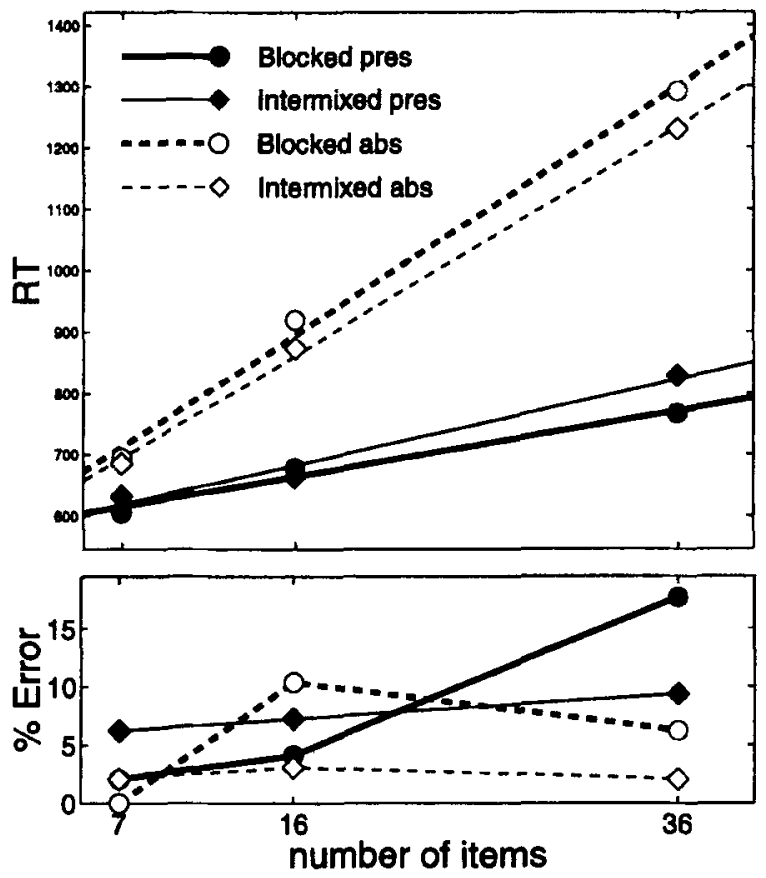

Figure 4. Mean reaction time (RT; top panel) and percent error (bottom panel) for blocked (circles) and intermixed (diamonds) presentation, collapsed across the three distractor pairs (in the relevant conditions, $B_{12}, B_{13}, B_{23}$, and $M_{12-12-23}$ ). Data from targetpresent trials are plotted with filled symbols; data from targetabsent trials are plotted with unfilled symbols. The regression lines are solid for target-present data and dashed for targetabsent data; they are thick for blocked presentation and thin for intermixed presentation. Observer E.O. 

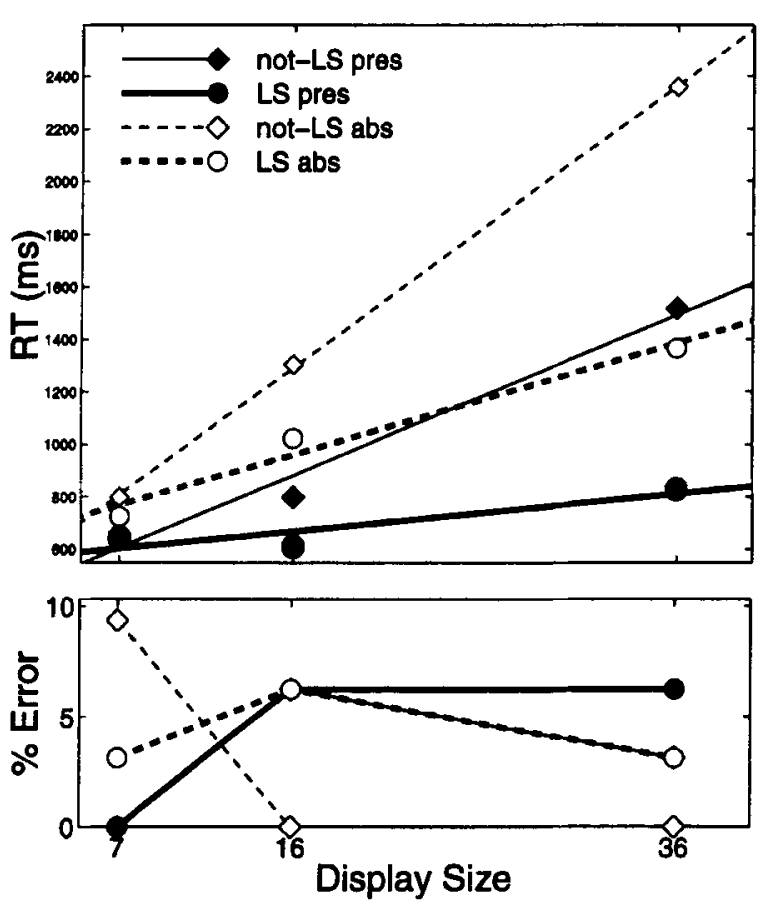

Figure 5. Mean reaction time (RT; top panel) and percent error (bottom panel) for Condition $B_{123}$ (not linearly separable; plotted as diamonds) and Condition $B_{124}$ (linearly separable; plotted as circles). The regression lines are solid for target-present data and dashed for target-absent data; they are thick for linearly separable displays and thin for displays that are not linearly separable. Observer E.O.

size was not significant $\left[F(4,1596)=.63, M S_{\mathrm{e}}=66,876\right.$, $p>.6]$, we collapsed across distractor pair for our calculation of slope differences between blocked and intermixed presentation. The comparison of blocked versus intermixed presentation yielded a small but significant main effect of $49 \mathrm{msec}\left[F(1,1608)=11.51, M S_{\mathrm{e}}=81,098\right.$, $p<.001]$ and no interaction with set size $[F(2,1608)=$ $.22, M S_{\mathrm{e}}=81,098, p>.8 \mathrm{]}$.

As for Observer E.O., for Observer S.L. individually presented pairs of the three distractors produced mueh faster search than did the triplet of distractors presented simultaneously $\left[F(1,1055)=95.33, M S_{\mathrm{e}}=122,357, p<\right.$ $.0001]$; the interaction of condition and set size was also significant $\left[F(2,1055)=19.64, M S_{\mathrm{e}}=122,357, p<\right.$ $.0001]$. Again, the difficulty of $\mathrm{B}_{123}$ search was not due to the number of distractor colors: $\mathrm{B}_{124}$ search was faster than $\mathrm{B}_{123}$ search $\left[F(1,526)=8.08, M S_{\mathrm{e}}=162,401, p<.005\right]$, and the interaction of condition and set size was significant $\left[F(2,526)=4.66, M S_{\mathrm{e}}=162,401, p<.01\right]$.

\section{GENERAL DISCUSSION}

The main conclusion from our data is that pop-out search is preserved in intermixed presentation of distractor pairs, even though the observer does not know in advance what classifier will be needed for a trial. We conclude this because we did not see a change in slope for intermixed presentation (which requires different search classifiers) relative to blocked presentation (where the same classifier can be used on every trial). The null slope difference across the mixed versus blocked conditions is unlikely to be due to a lack of statistical power because we were able to observe significant slope differences across the $B_{123}$ and $B_{124}$ conditions, which contrast easy and difficult search directly. Surprisingly, although the observer cannot use two linear separators simultaneously, he/she can quickly activate one of at least three different separators. It is clear that such flexibility is ecologically useful.

This result is an impressive extension of earlier findings associated with linear separability (Bauer et al., 1996b; D'Zmura, 1991). Even though the linear separator cannot be programmed in advance of stimulus presentation, the linear separability of the target from the (changing) distractors is nonetheless sufficient to support pop-out. There are at least two possible interpretations of this result. One is that the observers prepared three separators in advance and were able to activate the correct one. Another possibility is that an appropriate separator function can be constructed very quickly on the basis of bottom-up information alone. More research will be required to distinguish between these two possibilities.

Does this flexibility incur a constant cost? We found a cost for intermixed presentation relative to blocked presentation of $29 \mathrm{msec}$ (not significant) for Observer E.O. and of $49 \mathrm{msec}$ (significant) for Observer S.L. What does this cost represent? To perform the search task, the vi-

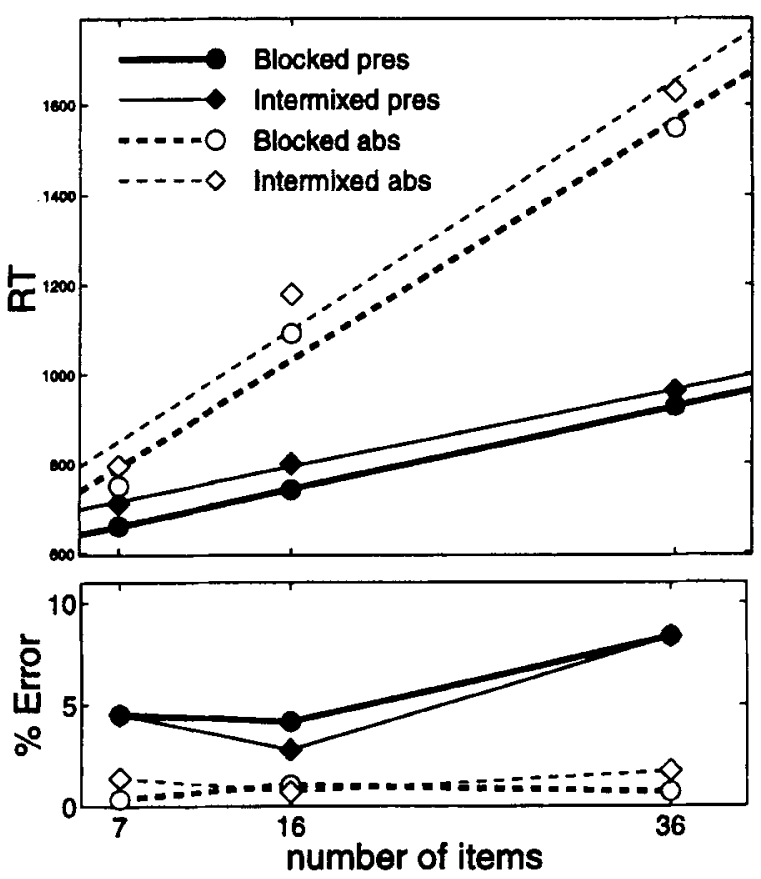

Figure 6. Mean reaction time (RT) and percent error for blocked (circles) and intermixed (diamonds) presentation, collapsed across the three distractor pairs. Observer S.L. 

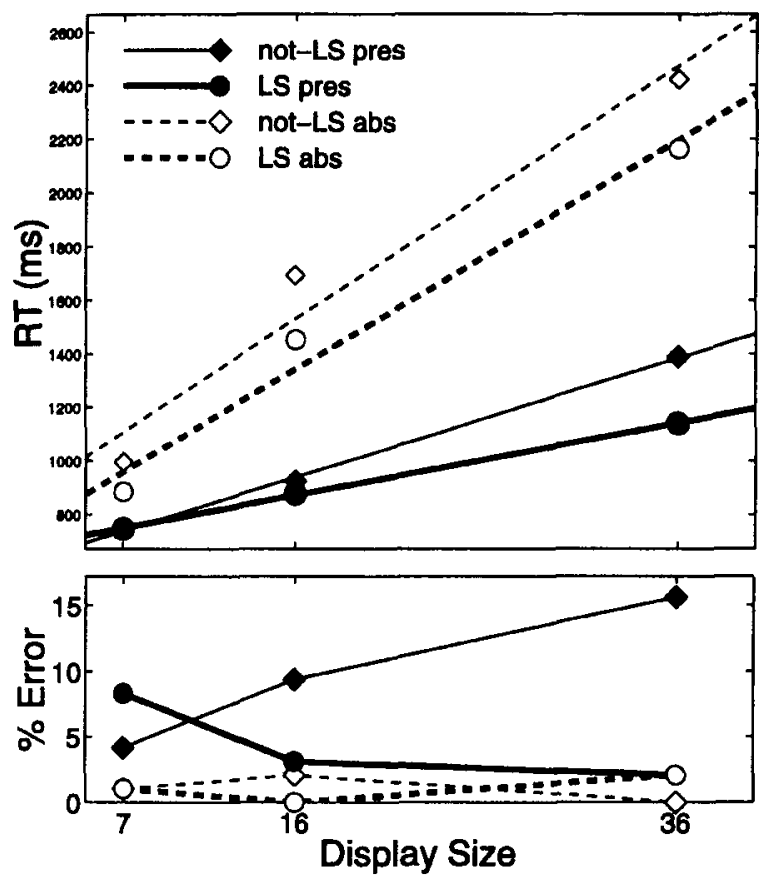

Figure 7. Mean reaction time (RT) and percent correct for Condition $B_{123}$ (not linearly separable [L5], diamonds) and Condition $B_{124}$ (linearly separable, circles), Observer S.L.

sual system must arrange a mapping to detect the target among the distractors that have appeared on the current trial, across multiple locations in the relevant portion of the visual field. In blocked presentation, this mapping can be created once for all trials, but in intermixed presentation it must be created or activated on a trial-by-trial basis. That the creation or activation of the correct mapping takes less than $50 \mathrm{msec}$ is quite impressive. This minimal cost suggests that stimulus-driven activation of the appropriate search classifier is a cognitively inexpensive operation. For comparison, consider that with an inefficient search slope of $30 \mathrm{msec} /$ item (Observer E.O.), this is less than the time it takes to search two items (assuming serial search). In addition, Olds, Cowan, and Jolicoeur (1998) have demonstrated that efficient search can be completed within $200 \mathrm{msec}$ of stimulus presentation, when target and distractor colors are known in advance; if stimulus uncertainty were to add to processing time less than a quarter of this duration, powerful guidance of vision could occur in less than a quarter of a second.

Several other studies have examined stimulus-driven effects in search. Zohary and Hochstein (1989) varied the ratio of the numbers of different distractor types from trial to trial. That is, in search for a red horizontal (conjunction) target, the display contained different numbers of red vertical distractors (as opposed to green horizontal distractors; the total number of distractors was constant across trials). Zohary and Hochstein found that observers' search strategy differed on the basis of this distractor ratio (which was not known in advance of a trial).
Observers searched only a subset of distractors for the target, and the results indicated that they chose to search the distractors that were less numerous (or rather, to not search the distractors that were more numerous). Given that observers could attain $70 \%$ correct performance with only 150-300 msec stimulus exposure, we can conclude that this guidance of search strategy occurred rather rapidly. Bacon and Egeth (1997) on the other hand, demonstrated that top-down information plays a role in this guidance by showing that observers performed more poorly when they were misinformed about distractor ratio.

Bravo and Nakayama (1992) also found stimulusdriven criterion formulation to be inexpensive, with a cost similar to the one we found, even though their task was quite different. We focus here on their target detection task (Experiment 1), in which observers had to detect a red target from among green distractors, or a green target from among red distractors. In the variable-mapping condition, the target and (homogeneous) distractors switched color (red vs. green) unpredictably from trial to trial, while in the consistent-mapping condition the assignment of color to target and distractor stimuli was blocked. Consistent with our results, Bravo and Nakayama found that the uncertainty caused by variability in mapping did not introduce a set-size effect-pop-out processes could perform the task. Instead, across all set sizes the variable-mapping RTs were approximately $40 \mathrm{msec}$ slower than the consistent-mapping RTs. Bravo and Nakayama's stimuli were different from ours in several ways, most importantly their distractors were homogeneous; ${ }^{4}$ however, it is noteworthy that they found uncertainty to cause a constant cost rather than a slope change, and that this constant cost was small. Other researchers have investigated the bottom-up power of singletons (unique stimuli) to capture attention even when it would be more adaptive for the observer to ignore them (see Egeth \& Yantis, 1997, for a review); these are examples of the power of singletons to mis-guide search in bottomup fashion.

However, the pop-out we found did not necessarily occur in the same way as in classic singleton search in homogeneous distractors. With detection in homogeneous distractors, the target is present if more than one color appears in the display - that is, if there is any contrast or discontinuity within the display items. In Bravo and Nakayama's (1992) experiment, RTs were around $400 \mathrm{msec}$, and this speed reflects the ease of this simple discontinuity detection. In our experiment, on the other hand, the distractors were not homogeneous. Mean search time in even the fastest conditions was around $600 \mathrm{msec}$. Target detection cannot be accomplished by simple discontinuity detection: Even when the target is not present, there is color contrast within the set of distractor items. ${ }^{5}$

The difference in difficulty between search in homogeneous and heterogeneous distractors is also particularly relevant in displays with small set sizes. In our experiment, as few as two of each distractor color were presented in the seven-item displays of the distractor- 
triple conditions ( $\mathrm{B}_{123}$ and $\mathrm{B}_{124}$ ). It is likely that in our experiment the consistent target color provided goal-driven input to search processes, along with the stimulus-driven input required for performance in intermixed presentation.

Further research will be necessary to explore a variety of types of uncertainty. It is possible to intermix trials on which the target is linearly separable from distractors with trials on which the target is not linearly separable. It is also possible to manipulate certainty about target characteristics as well as uncertainty about distractor characteristics in order to completely remove the effect of top-down knowledge of target color, for example.

Perception is guided by the interaction of goals and stimuli. We found the stimulus to be powerful enough to direct the search process in an impressively small amount of time. This direction allowed easy search mechanisms to perform the intermixed task, even though no classifier could be preselected for use on all trials. Although multiple classifiers cannot be used simultaneously on a given display, multiple classifiers can be activated for use at low cost.

\section{REFERENCES}

BACON, W. F., \& EGETH, H. E. (1997). Goal-directed guidance of attention: Evidence from conjunctive visual search. Journal of Experimental Psychology: Human Perception \& Performance, 23, 948-961.

Bauer, B., Jolicoeur, P., \& Cowan, W. B. (1996a). Distractor heterogeneity versus linear separability in colour visual search. Perception, 25, 1281-1293.

Bauer, B., Jolicoeur, P., \& Cowan, W. B. (1996b). Visual search for colour targets that are or are not linearly-separable from distractors. Vision Research, 36, 1439-1466.

Brainard, D. H. (1997). The Psychophysics Toolbox. Spatial Vision, $10,433-436$.

Bravo. M. J.. \& Nakayama, K. (1992). The role of attention in different visual-search tasks. Perception \& Psychophysics, 51, 465-472.

DUNCAN, J., \& Humphreys, G. W. (1989). Visual search and stimulus similarity. Psychological Review, 96, 433-458.

D'Zmura, M. (1991). Color in visual search. Vision Research, 31, 951966.

EgETh, H. E., \& YANTIS. S. (1997). Visual attention: Control, representation, and time course. Annual Review of Psychology, 48, 269-297.

Olds, E. S., Cowan, W. B., \& Jolicoeur. P. (1999). Tracking visual search over space and time. Manuscript submitted for publication.

ROSENHOLTZ, R. (1999). A simple saliency model predicts a number of motion popout phenomena. Vision Research, 39, 3157-3163.
Treisman, A. M., \& Gelade, G. (1980). A feature-integration theory of attention. Cognitive Psychology, 12, 97-136.

VAN SElst, M., \& Jolicoeur, P. (1994). A solution to the effect of sample size on outlier elimination. Quarterly Journal of Experimental Psychology, 47A, 631-650.

Wyszecki, G., \& Stiles, W. S. (1982). Color science: Concepts and methods, quantitative data and formulae (2nd ed.). New York: Wiley.

ZohaRY, E., \& HochSTEIN, S. (1989). How serial is serial processing in vision? Perception, 18, 191-200.

\section{NOTES}

1. Effects similar to linear separability have been demonstrated in dimensions besides color. For example, Rosenholtz (1999) explained differences in the saliency of motion targets by examining the distribution of distractor velocities and calculating the distance between target velocity and mean distractor velocity, in units of distractor velocity. In many cases the predictions of this model are the same as those that would be generated by linear separability in velocity space, but in addition they provide further quantitative explanations of some effects of distractor heterogeneity. It is possible that search mechanisms can be rapidly determined by the stimulus in these other dimensions as well.

2. Observer E.O. had normal color vision as assessed by Ishihara color plates and Nagel anomaloscope; Observer S.L. had normal color vision as assessed by Ishihara plates.

3. We concluded this by regressing RT on blocked/intermixed and set size to determine the coefficient and $t$ statistic associated with the factor blocked/intermixed. The coefficient was 18 ; the $t$ statistic was 1.06 . The factor by which the obtained $t$ statistic would need to be multiplied to obtain significance $(t$ of 1.96 would be significant at the .05 level with infinite degrees of freedom) was $1.96 / 1.06=1.85$. We multiplied the coefficient we obtained in the regression $(18 \mathrm{msec})$ by this factor to estimate the size the main effect would need to be, given the noise in the data, to reach significance.

4. In addition, in their experiment both target and distractors changed color. Therefore, if the linear separator is a plane through color space detecting contrast only, then the same linear separator could segregate target from distractors in color space for all trials even under uncertainty; if the linear separator function includes the sign of the contrast (i.e., if the sign has to be positive on the side of the target), then two separators would be required. Also, the color differences between their stimuli were greater than the color differences in our experiment. Finally, Bravo and Nakayama (1992) used a black background, not an isoluminant gray background.

5. To put it another way, in our two-distractor-colors tasks, the observer had to differentiate between two colors (target absent) and three colors (target present) in the display. This may be more difficult than differentiating between one and two colors, as in the Bravo and Nakayama (1992) task.

(Manuscript received April 7, 1998; revision accepted for publication July $28,1998$. 